\title{
Focused ultrasound-induced blood-brain barrier opening improves adult hippocampal neurogenesis and cognitive function in a cholinergic degeneration dementia rat model
}

Jaewoo Shin ${ }^{1,2+}$, Chanho Kong ${ }^{1+}$, Jihyeon Lee ${ }^{1,2}$, Bo Young Choi ${ }^{3}$, Jiyeon Sim ${ }^{1,2}$, Chin Su Koh ${ }^{1}$, Minkyung Park ${ }^{1,2}$, Young Cheol $\mathrm{Na}^{4}$, Sang Won Suh ${ }^{3}$, Won Seok Chang ${ }^{1 *}$ and Jin Woo Chang ${ }^{1,2^{*}}$ (i)

\begin{abstract}
Background: The persistence of adult hippocampal neurogenesis (AHN) is sharply decreased in Alzheimer's disease (AD). The neuropathologies of AD include the presence of amyloid- $\beta$ deposition in plaques, tau hyperphosphorylation in neurofibrillary tangles, and cholinergic system degeneration. The focused ultrasound (FUS)mediated blood-brain barrier opening modulates tau hyperphosphorylation, the accumulation of amyloid- $\beta$ proteins, and increases in AHN. However, it remains unclear whether FUS can modulate AHN in cholinergicdeficient conditions. In this study, we investigated the effect of FUS on AHN in a cholinergic degeneration rat model of dementia.
\end{abstract}

Methods: Adult male Sprague-Dawley rats $(n=48 ; 200-250 \mathrm{~g}$ ) were divided into control (phosphate-buffered saline injection), $192 \mathrm{lgG}$-saporin (SAP), and SAP+FUS groups; in the two latter groups, SAP was injected bilaterally into the lateral ventricle. We applied FUS to the bilateral hippocampus with microbubbles. Immunohistochemistry, enzyme-linked immunosorbent assay, immunoblotting, 5-bromo-2'-deoxyuridine labeling, an acetylcholinesterase assay, and the Morris water maze test were performed to assess choline acetyltransferase, acetylcholinesterase activity, brain-derived neurotrophic factor expression, neural proliferation, and spatial memory, respectively. Statistical significance of differences in between groups was calculated using one-way and two-way analyses of variance followed by Tukey's multiple comparison test to determine the individual and interactive effects of FUS on immunochemistry and behavioral analysis. $P<0.05$ was considered significant.

Results: Cholinergic degeneration in rats significantly decreased the number of choline acetyltransferase neurons $(P<0.05)$ in the basal forebrain, as well as AHN and spatial memory function. Rats that underwent FUS-mediated brain-blood barrier opening exhibited significant increases in brain-derived neurotrophic factor (BDNF; $P<0.05$ ), early growth response protein 1 (EGR1) $(P<0.01)$, AHN $(P<0.01)$, and acetylcholinesterase activity in the frontal cortex $(P<0.05)$ and hippocampus $(P<0.01)$ and crossing over $(P<0.01)$ the platform in the Morris water maze relative to the SAP group after sonication.

(Continued on next page)

\footnotetext{
* Correspondence: changws0716@yuhs.ac; jchang@yuhs.ac

${ }^{\dagger}$ Jaewoo Shin and Chanho Kong contributed equally to this work.

${ }^{1}$ Department of Neurosurgery, Yonsei University College of Medicine, Seoul

03722, Republic of Korea

Full list of author information is available at the end of the article
}

(c) The Author(s). 2019 Open Access This article is distributed under the terms of the Creative Commons Attribution 4.0 International License (http://creativecommons.org/licenses/by/4.0/), which permits unrestricted use, distribution, and reproduction in any medium, provided you give appropriate credit to the original author(s) and the source, provide a link to the Creative Commons license, and indicate if changes were made. The Creative Commons Public Domain Dedication waiver (http://creativecommons.org/publicdomain/zero/1.0/) applies to the data made available in this article, unless otherwise stated. 
(Continued from previous page)

Conclusions: FUS treatment increased AHN and improved spatial memory. This improvement was mediated by increased hippocampal BDNF and EGR1. FUS treatment may also restore AHN and protect against neurodegeneration, providing a potentially powerful therapeutic strategy for AD.

Keywords: Rats, Sprague-Dawley, Brain-derived neurotrophic factor, Microbubbles, Sonication, Hippocampus, Dementia, Alzheimer's disease, Neuropathology,

\section{Background}

Adult neurogenesis appears to be restricted to two regions, i.e., the subventricular zone (SVZ) of the lateral ventricles and the subgranular zone (SGZ) of the hippocampal dentate gyrus (DG). Importantly, adult hippocampal neurogenesis $(\mathrm{AHN})$ was first reported over 50 years ago by Altman and Das [1], and newborn neurons are generated continuously throughout life in the mammalian brain, including the human brain [2, 3]. Since then, numerous studies have reported that AHN is implicated in cognition and endogenous repair mechanisms in normal physiological conditions such as learning and memory [4]. Interestingly, according to the recent research, the persistence of AHN appears to be decreased in aged adults and Alzheimer's disease (AD) $[5,6]$.

$\mathrm{AD}$ is one of the major causes of age-related dementia and is characterized by cognitive impairment, amyloid- $\beta$ deposition in plaques, tau hyperphosphorylation in neurofibrillary tangles, loss of synapses, loss of neuronal cells, and cholinergic dysfunction [7]. Dysfunction of the basal forebrain cholinergic (BFC) system, a significant characteristic of $\mathrm{AD}$, induces neuropathological changes before clinical symptoms manifest [8-10]. The hippocampus and cortex receive gamma-aminobutyric acidergic, glutamatergic, and cholinergic input from the basal forebrain of the medial septum-diagonal band complex (MS/DB) $[11,12]$. Thus, lesions in, or the inactivation of, cholinergic neurons in $\mathrm{MS} / \mathrm{DB}$ result in a decrease of acetylcholinesterase (AChE) and choline acetyltransferase (ChAT), consequently diminishing AHN [13-16].

Despite intensive research efforts, none of the currently available treatments for $\mathrm{AD}$ can completely cure or prevent the course of age-related cognitive impairment, and the pathological mechanism is not clearly understood. Numerous pharmacological therapies have been developed to treat AD [17]. However, 98\% of small-molecule drugs $(<400 \mathrm{Da})$ and $100 \%$ of largemolecule drugs (>500 Da) cannot cross the blood-brain barrier (BBB) [18], making the prevention and treatment of brain disorders difficult.

Focused ultrasound (FUS) combined with contrast agent microbubbles is a noninvasive technique that transiently opens BBB in targeted regions, thereby enabling localized therapeutic drug, gene, or nanoparticle delivery into the brain for treating central nervous system (CNS) disorders [19-21]. Considering that drugs that have been, or are currently being, developed for $\mathrm{AD}$ are mostly large molecules, FUS may enhance the effects of these drugs especially in patients with early-stage AD who have an intact BBB [22]. Moreover, several reports suggest that FUS stimulates neuronal activity and modulates proteomes and transcriptomes, independent of any therapeutic agent [23-25].

Previous studies indicate that FUS-mediated BBB opening can modulate the accumulation of amyloid- $\beta$ and tau hyperphosphorylation in AD transgenic mice and increase AHN in wild-type mice [26-30]. Recently, Moreno-Jiménez et al. reported the persistence of AHN in human DG of subjects aged over 90 years; however, the number and maturation of immature neurons in DG sharply decreased in patients with $\mathrm{AD}$. This finding has gained attention for potential therapeutic strategies as an underlying memory impairment in $\mathrm{AD}$ [31]. However, it remains unclear whether FUS can modulate AHN in a cholinergic-deficient condition. In this study, we investigated the effect of FUS on AHN and the cholinergic system in a cholinergic degeneration dementia rat model, which is a key pathogenic feature of dementia. Furthermore, if FUS was effective in increasing AHN, the synergistic effects of AHN modulation and drug delivery could improve treatment outcomes of AD.

\section{Materials and methods}

Ethical considerations

All animal experimental procedures were conducted in compliance with the Guide for the Care and Use of Laboratory Animals of the National Institutes of Health and were approved by the Institutional Animal Care and Use Committee (IACUC; 2016-0339) of Yonsei University. Animals were housed in groups of three in laboratory cages with food and water available ad libitum in a 12-h light/dark (lights on at 07:00) cycle in a room with controlled temperature $\left(22 \pm 2{ }^{\circ} \mathrm{C}\right)$ and humidity $(55 \pm$ $5 \%)$.

\section{Rat model generation}

Previous studies have modeled cholinergic degeneration and cognitive function-impaired dementia in rats by intraventricularly administering the selective immunotoxin 192 IgG-saporin (SAP) to induce lesions in BFC neurons 
[32-35]. To investigate the effect of FUS on AHN in a cholinergic degeneration rat model of dementia, adult male Sprague Dawley rats $(n=48 ; 200-250 \mathrm{~g})$ were divided into control (phosphate-buffered saline [PBS] injection), SAP, and SAP+FUS groups. The dementia rat model (SAP, $n=16$; SAP+FUS, $n=16$ ) was generated by injecting SAP (Chemicon, Temecula, CA, USA), and the control group $(n=16)$ received a bilateral ventricular infusion of $1 \times$ PBS (PH 7.4) into the brain. All 48 rats were anesthetized with a mixture of ketamine $(75 \mathrm{mg} /$ $\mathrm{kg}$ ), xylazine (Rompun ${ }^{\mathrm{Tw}} ; 4 \mathrm{mg} / \mathrm{kg}$ ), and acepromazine $(0.75 \mathrm{mg} / \mathrm{kg})$ and were fixed in a stereotaxic frame. As previously described, scalp skin was incised, and two holes were drilled into the skull at the following coordinates: from the bregma anterior-posterior, $-0.8 \mathrm{~mm}$; medial-lateral, $\pm 1.2 \mathrm{~mm}$; and dorsal-ventral, $-3.4 \mathrm{~mm}$ [36]. Thereafter, $4 \mu \mathrm{l}$ of SAP $(0.63 \mu \mathrm{g} / \mu \mathrm{l})$ was bilaterally injected at a rate of $1 \mu \mathrm{l} / \mathrm{min}$ into the lateral ventricle of the rats in the SAP and SAP+FUS groups using a syringe pump (Legato 130, 788130, KD Scientific, Holliston, MA, USA). As shown in Fig. 1a, rats were sacrificed at different time points, i.e., $24 \mathrm{~h}, 5$ days, and 18 days after FUS. To detect changes in AChE and BDNF expression levels, observe proliferation and neuroblast production, and observe neuronal differentiation of BrdU-positive cells and long-term effects of AChE and BDNF, the rats were sacrificed $24 \mathrm{~h}, 15$ days, and 18 days after FUS, respectively.

\section{Focused ultrasound}

The pulsed ultrasound was generated using a $0.5-\mathrm{MHz}$ single-element spherically focused transducer (H-
107MR, Sonic Concept Inc., Bothell, WA, USA) with a diameter of $51.7 \mathrm{~mm}$ and radius of curvature of 63.2 $\mathrm{mm}$. A waveform generator (33220A, Agilent, Palo Alto, CA, USA) was connected to a 50-dB Radio Frequency Power Amplifier (240 L, ENI Inc., Rochester, NY, USA) to drive the FUS transducer, and a power meter (E4419B, Agilent) was used to measure the input electrical power. The transducer electrical impedance was matched to the output impedance of the amplifier $(50 \Omega)$ with an external matching network (Sonic Concept Inc., Bothell, WA, USA). A cone filled with distilled, degassed water was mounted onto the transducer assembly (Additional file 1: Figure S1). A needle-type hydrophone (HNA-0400, Onda, Sunnyvale, CA, USA) was used for the transducer calibration, which measured the acoustic beam profile in the tank filled with degassed water. The transducer was mounted on the cone filled with degassed water, and the end of its tip was wrapped in a polyurethane membrane.

The experimental procedure is shown in Fig. 1. Briefly, rats were anesthetized with a mixture of ketamine (75 $\mathrm{mg} / \mathrm{kg}$ ) and xylazine $(4 \mathrm{mg} / \mathrm{kg})$, and their heads mounted on a stereotaxic frame (Narishige, Tokyo, Japan) with ear and nose bars. Ultrasound transmission gel (ProGelDayo Medical Co., Seoul, South Korea) was used to cover the area between the animal's skull and the cone tip to maximize the transmission efficiency of the ultrasound. FUS was targeted bilaterally to the region containing the hippocampus according to the 3D positioning system. DEFINITY ${ }^{\circ}$ microbubble contrast agents (mean diameter range, $1.1-3.3 \mu \mathrm{m}$; Lantheus Medical Imaging, North Billerica, MA, USA) were

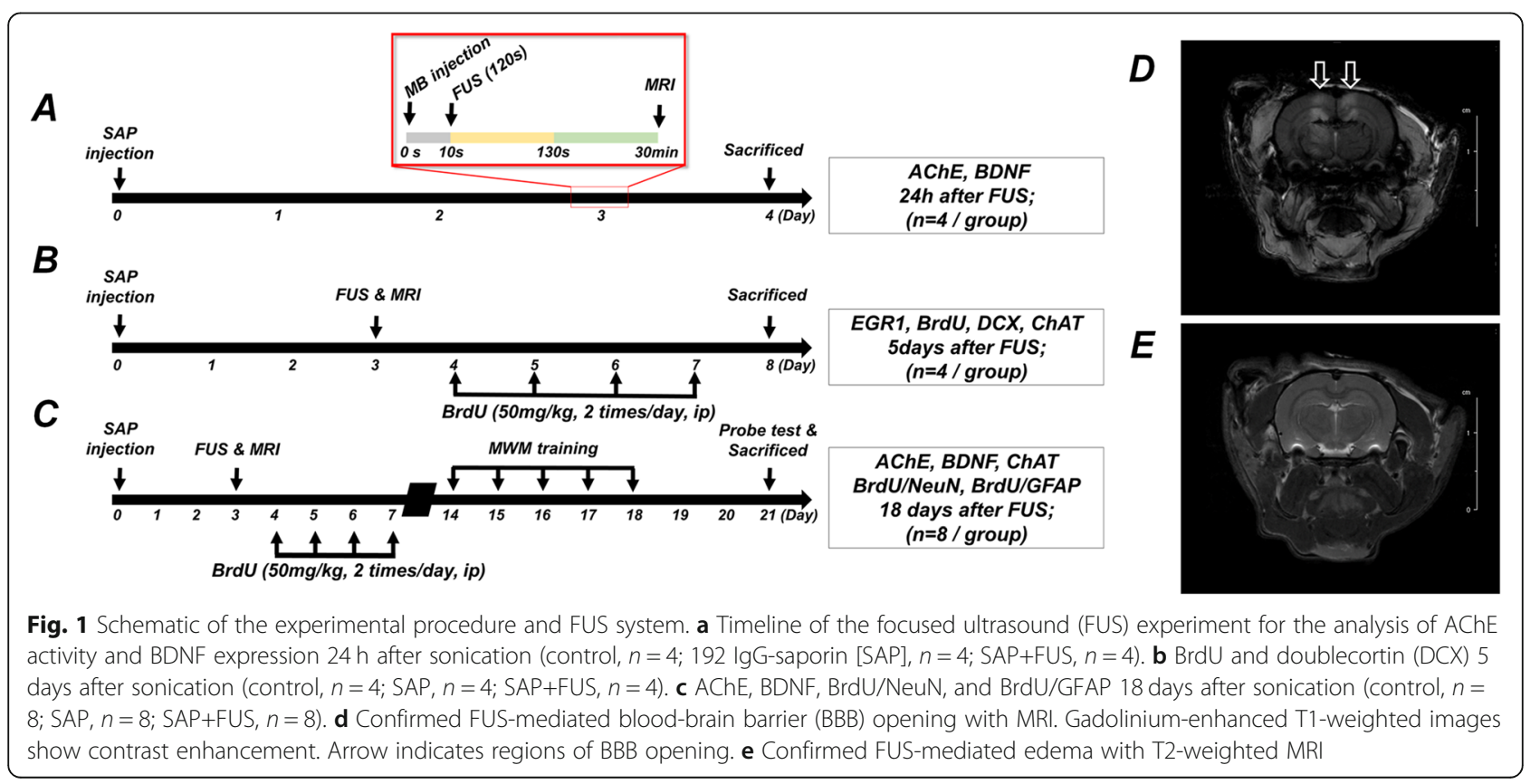


diluted in saline and injected intravenously into the tail vein $10 \mathrm{~s}$ before sonication. Sonication parameters were set as follows: burst duration, $10 \mathrm{~ms}$; pulse repetition frequency, $1 \mathrm{~Hz}$; total duration, $120 \mathrm{~s}$; and average peaknegative pressure, $0.25 \mathrm{MPa}$.

\section{Magnetic resonance imaging}

After sonication, magnetic resonance imaging (MRI) experiments were performed with a Bruker $9.4 \mathrm{~T} 20-\mathrm{cm}$ bore MRI system (Biospec 94/20 USR; Bruker, Ettlingen, Germany) and a rat head coil. A gadolinium-based contrast agent, gadobutrol (Gd, Gadovist; Bayer Schering Pharma AG, Berlin, Germany; $0.2 \mathrm{~mL} / \mathrm{kg}$ ), was injected into the tail vein, and contrast-enhanced T1-weighted images were used to confirm the BBB opening from the FUS. T1-weighted MRI was performed with and without the use of gadobutrol contrast (Fig. 1d). T2-weighted images were used to confirm edema with FUS (Fig. 1e). Sequence parameters are summarized in Table 1.

\section{Behavioral test-Morris water maze}

Rats underwent the Morris water maze (MWM) test at 2 weeks after receiving SAP injection. The MWM apparatus comprised a circular pool (diameter, $2 \mathrm{~m}$; height, $50 \mathrm{~cm})$ filled to a depth of $30 \mathrm{~cm}$ with dark water $\left(23^{\circ} \mathrm{C}\right)$. A concealed black, round platform (diameter, $15 \mathrm{~cm}$ ) was situated $1-2 \mathrm{~cm}$ below the surface of the water in the center of a target quadrant. All rats were trained for four trials per day for 5 consecutive days. During training, the location of the hidden platform was fixed, and spatial cues were provided for guidance. For each training trial, the rats were placed in the water facing the wall at one of the four starting points and were given $60 \mathrm{~s}$ to reach the hidden platform. After finding the platform, the rats were allowed to remain on the platform for $10 \mathrm{~s}$. The rats that could not find the platform within $60 \mathrm{~s}$ were led to the platform by the experimenter and were allowed to remain on the platform for $10 \mathrm{~s}$. The rats were given a 60-s probe test without the platform $72 \mathrm{~h}$ after the last training trial. Swimming speed, swim path, time spent in each zone, and distance swam were

Table 1 Sequences and parameters of MRI

\begin{tabular}{lll}
\hline & T1-weighted Imaging & T2-weighted Imaging \\
\hline Echo & 1 & 1 \\
TR (ms) & 350 & 2500 \\
TE (ms) & 5.4 & 33 \\
FA (deg) & 40 & 180 \\
NEX & 2 & 2 \\
FOV (cm) & 3.5 & 3.5 \\
Matrix & $256 \times 256$ & $256 \times 256$ \\
\hline
\end{tabular}

TR repetition time, TE time to echo, FA fractional anisotropy, NEX number of excitations, FOV field of view recorded using the SMART video-tracking system (Harvard Apparatus, Holliston, MA, USA).

\section{BrdU labeling}

To investigate the effect of FUS on neurogenesis, animals were injected intraperitoneally with 5-bromo-2' deoxyuridine (BrdU; Sigma-Aldrich, St. Louis, MO, USA), used for the detection of proliferating cells, twice a day for 4 consecutive days, $24 \mathrm{~h}$ after sonication [30, 37].

\section{Histological evaluation \\ Brain tissue preparation}

The animals were sacrificed 5 days $(n=4$ per group) or 18 days ( $n=4$ per group) after FUS sonication. The rats were anesthetized via the intraperitoneal injection of a mixture of ketamine $(75 \mathrm{mg} / \mathrm{kg})$ and xylazine $(4 \mathrm{mg} / \mathrm{kg})$. For the blood wash-out and brain fixation, transcranial perfusion was performed with $0.9 \%$ normal saline and $4 \%$ paraformaldehyde in $1 \times$ PBS. After perfusion, all brains were post-fixed in $4 \%$ paraformaldehyde for $1 \mathrm{~h}$. Subsequently, the brain tissue was transferred to a $30 \%$ sucrose solution for 3 days. The brains were then sectioned into 30- $\mu$ m-thick slices using a Leica CM1850 cryostat (Leica Biosystems, Wetzlar, Germany).

\section{Immunohistochemistry}

To determine the effects of FUS on cell proliferation, 24 $\mathrm{h}$ after sonication, brain sections were incubated in $0.3 \%$ $\mathrm{H}_{2} \mathrm{O}_{2}$ for $15 \mathrm{~min}$ to inactivate endogenous peroxidase activity. DNA denaturation was then performed by antigen retrieval in $2 \mathrm{~N} \mathrm{HCl}$ at $37^{\circ} \mathrm{C}$ for $90 \mathrm{~min}$ and neutralization twice with $0.1 \mathrm{M}$ borate buffer for $10 \mathrm{~min}$. The sections were then washed with PBS, blocked with $5 \%$ normal goat serum for $1 \mathrm{~h}$, and incubated overnight at $4{ }^{\circ} \mathrm{C}$ with the following monoclonal antibodies diluted in PBS containing 0.3\% normal goat serum and $0.3 \%$ Triton X-100: mouse anti-BrdU (1:150, BMC9313, Roche Molecular Biochemicals, Mannheim, Germany), rabbit anti-early growth response 1 antibody (EGR1; 1 : 200, 4153S, Cell Signaling Technology, Inc., Beverly, MA, USA), goat anti-DCX (1:200, SC8066, Santa Cruz Biotechnology, Inc., Santa Cruz, CA, USA), and goat anti-ChAT (1:100, AB144P, Millipore, Bedford, MA, USA). Thereafter, the sections were incubated with affinity-purified biotinylated goat anti-mouse IgG secondary antibodies (1:400, BA-9200, Vector Laboratories, Burlingame, CA, USA), affinity-purified biotinylated rabbit anti-goat IgG secondary antibodies (1:400, BA5000, Vector Laboratories, CA, USA), affinity-purified biotinylated goat rabbit anti-IgG secondary antibodies (1:400, BA-1000, Vector Laboratories, CA, USA), and affinity-purified biotinylated according to the avidinbiotin complex method (ABC Elite; Vector Laboratories, 
CA, USA). Immunoreactivity was evaluated using a DAB substrate kit (Thermo Fisher Scientific, Fremont, CA, USA). EGR1 was counterstained using hematoxylin (H3401, Vector Laboratories, CA, USA). The samples were examined using an optic microscope (BX51; Olympus, Tokyo, Japan).

\section{Immunofluorescence staining}

The sections were double stained with BrdU and NeuN or BrdU and GFAP and then incubated for $2 \mathrm{~h}$ in a mixture of mouse monoclonal anti-BrdU (1:150, BMC9313, Roche Molecular Biochemicals, Mannheim, Germany) and either rabbit polyclonal anti-NeuN (Neuronal nuclei; 1:500, ABN78, Millipore, Bedford, MA, USA) or goat polyclonal anti-GFAP (Glial fibrillary acidic protein; AB7260 1:200, Abcam Cambridge, MA, USA). This was followed by a 2-h incubation in a mixture of goat antimouse Alexa Fluor ${ }^{\circledR} 594$ IgG (1:500, A11005, Invitrogen, Carlsbad, CA, USA) (BrdU) and goat anti-rabbit Alexa Fluor $^{\oplus}$ 488(1:500, A11008, Invitrogen, Carlsbad, CA, USA) (NeuN, GFAP) at room temperature. Fluorescence signals were confirmed using a Zeiss LSM 710 confocal imaging system (Carl Zeiss, Oberkochen, Germany) with a sequential scanning mode for Alexa 594 and 488. Stacks of images $(1024 \times 1024$ pixels $)$ from consecutive $0.9-1.2-\mu \mathrm{m}$-thick slices were obtained by averaging eight scans per slice. The resulting images were processed with ZEN 2010 (Carl Zeiss).

\section{Quantification of cell counting}

Seven coronal sections (185- $\mu \mathrm{m}$ intervals) from each animal, collected from 3.2 to $4.5 \mathrm{~mm}$ posterior to the bregma, were analyzed to quantify the BrdU-, DCX-, and EGR1-positive cells. The sections were photographed using a virtual microscope (BX51; Olympus) with $\mathrm{a} \times 10$ objective. Coded sections were counted by a blinded observer who quantified the number of BrdUand DCX-positive cells in the bilateral subgranular zone (SGZ) and granular cell layer (GCL) of the dentate gyrus (DG) and the number of EGR1-positive cells in the bilateral CA1, CA3, and DG of the hippocampus. To analyze the phenotype of BrdU-positive cells, we determined whether BrdU-positive cells in the SGZ and GCL (SGZ/ GCL) expressed NeuN or GFAP with confocal microscopy. A double-positive percentage was calculated as $\mathrm{BrdU}+/ \mathrm{NeuN}+$ or BrdU+/GFAP+ for total BrdU-positive cells in the SGZ/GCL.

\section{ELISA and immunoblotting}

\section{Brain sample preparation}

At $24 \mathrm{~h}(n=12)$ and 18 days $(n=12)$ after sonication, the remaining rats from each group were anesthetized with a mixture of ketamine $(75 \mathrm{mg} / \mathrm{kg})$, xylazine $(4 \mathrm{mg} / \mathrm{kg})$, and acepromazine $(0.75 \mathrm{mg} / \mathrm{kg})$. They were then decapitated with a guillotine, and their brains removed. The prefrontal cortex and hippocampus regions were dissected with fine forceps to yield 1-mm coronal brain slices using a rat brain slicer matrix. These samples were homogenized in a Kontes glass homogenizer (Kontes Glass Co., Vineland, NJ, USA) with a protein extraction solution containing 1.0 mM PMSF, 1.0 mM EDTA, $1 \mu \mathrm{M}$ pepstatin, $1 \mu \mathrm{M}$ leupeptin, and $1 \mu \mathrm{M}$ aprotinin (PROPREP, Catalog no. 17081, iNtRON Biotechnology, Seongnam, Korea). After extraction, the slices were centrifuged for $20 \mathrm{~min}$ at $12,000 \mathrm{rpm}$. The total protein concentration was measured using the bicinchoninic acid protein assay reagent kit (Pierce, Rockford, IL, USA). All extraction steps were performed at $4{ }^{\circ} \mathrm{C}$, and protein samples were stored at $-80^{\circ} \mathrm{C}$ until use.

\section{AChE assay}

To evaluate the enzymatic activity of AChE, the modified version of the method of Ellman et al. was used [38]. In brief, 20- $\mu$ l triplicate samples were mixed with a reaction mixture $\left(0.2 \mathrm{mM} 5,5^{\prime}\right.$-dithiobis (2-nitrobenzoic acid) [Sigma-Aldrich], $0.56 \mathrm{mM}$ acetylthiocholine iodide [Sigma-Aldrich], $10 \mu \mathrm{M}$ tetraisopropyl pyrophosphoramide [Sigma-Aldrich], and $39 \mathrm{mM}$ phosphate buffer; $\mathrm{pH}$ 7.2 ) at $37^{\circ} \mathrm{C}$ for $30 \mathrm{~min}$. The quantification of optical density was performed at a wavelength of $405 \mathrm{~nm}$.

\section{Western blot analysis}

Western blot analyses were performed on the same protein samples as those used for the AChE assay. Twenty micrograms of each protein were separated by $12 \%$ sodium-dodecyl-sulfate-polyacrylamide gels and electrotransferred onto polyvinylidene fluoride membranes using a Bio-Rad miniature transfer apparatus for 100 min at $0.3 \mathrm{~A}$. The membranes were then blocked using a blocking buffer (5\% non-fat dry milk in PBS containing $0.05 \%$ Tween 20$)$ for $1 \mathrm{~h}$ at room temperature $\left(25^{\circ} \mathrm{C}\right)$. The membranes were then incubated with primary antibodies overnight at $4{ }^{\circ} \mathrm{C}$ with rabbit monoclonal antibrain-derived neurotrophic factor (BDNF, 1:1000; Abcam, Cambridge, UK) and mouse monoclonal anti- $\$$ actin (1:10000; Sigma-Aldrich). The corresponding secondary antibodies were then applied for $90 \mathrm{~min}$ at room temperature with goat anti-rabbit $\operatorname{IgG}(\mathrm{H}+\mathrm{L})-\mathrm{HRP}$ (1: 2000 at BDNF; GenDEPOT, Katy, TX, USA) and goat anti-mouse IgG(H+L)-HRP (1:10000; GenDEPOT). The proteins were visualized using an enhanced chemiluminescence solution (WEST-Queen western blot detection kit, iNtRON Biotechnology), and blots were analyzed using a LAS 4000 mini (GE Healthcare Life Sciences). The intensity of each band was measured using optical densitometry of the analysis system (Multi Gauge version 3.0; Fujifilm, Tokyo, Japan). 


\section{Statistical analysis}

All data are expressed as mean \pm standard error of the mean. Statistical significance of differences between groups was calculated using one-way and two-way analysis of variance followed by Tukey's multiple comparisons test to determine the individual and interactive effects of FUS on immunochemistry and behavioral analysis. $P<0.05$ was considered significant. All statistical analyses were performed using SPSS (Version 20, SPSS Inc., Chicago, IL, USA) and GraphPad Prism 5 software (GraphPad Software Inc., San Diego, CA, USA).

\section{Results}

\section{Confirmation of cholinergic degeneration by SAP}

To confirm cholinergic degeneration in our model, we quantified ChAT-immunopositive cells in MS/DB of each group of rats. Five days after sonication, compared with the control group $(100 \pm 3.5 \%)$, both the SAP $(65.5 \pm 13.1 \% ; \quad P<0.05)$ and SAP+FUS (48.6 \pm 5.05 ; $P<0.01)$ groups displayed a significantly reduced number of ChAT-immunopositive neurons (Fig. 2b). Eighteen days after sonication, compared with the control group $(100 \pm 10)$, both the SAP $(18.7 \pm 4.3 ; P<0.001)$ and SAP+FUS $(13.89 \pm 5.9 ; P<0.001)$ groups had significantly fewer ChAT-immunopositive neurons and less neuronal damage to cholinergic neuron bodies (Fig. 2c).
These results indicate that the number of cholinergic neurons were decreased in all groups at 5 and 18 days, which provide supporting evidence that dementia model using SAP was effective.

\section{FUS affects AChE activity in a dementia rat model}

To determine whether FUS affects cholinergic neuronal activity, we quantified AChE activity in each group. Twenty-four hours after sonication, AChE activity was significantly reduced in the SAP group in the frontal cortex (FC; $68.61 \pm 3.02 \% ; P<0.05$ ) and hippocampus $(86.12 \pm 1.43 \% ; P<0.05)$ compared with that in the control group (Fig. 3a, b).

Eighteen days after sonication, AChE activity was significantly decreased in the SAP group in the FC $(74.85 \pm$ $3.62 \% ; P<0.05)$ and hippocampus $(83.70 \pm 1.61 \% ; P<$ 0.01 ) compared with that in the control group (Fig. 3c, d). However, the AChE activity of the hippocampus was significantly increased in the SAP+FUS group (94.03 \pm $2.33 \% ; P<0.01)$ compared with that in the SAP group. The AChE activity of the FC was increased in the SAP+FUS group $(90.79 \pm 5.30 \% ; P=0.09)$ compared with that in the SAP group, but there was no significant difference between the two groups (Fig. 3c, d).

As a result, cholinergic degeneration of MS induced decreased activities of AChE in both FC and hippocampus at $24 \mathrm{~h}$ and 18 days. The effect of FUS treatment in

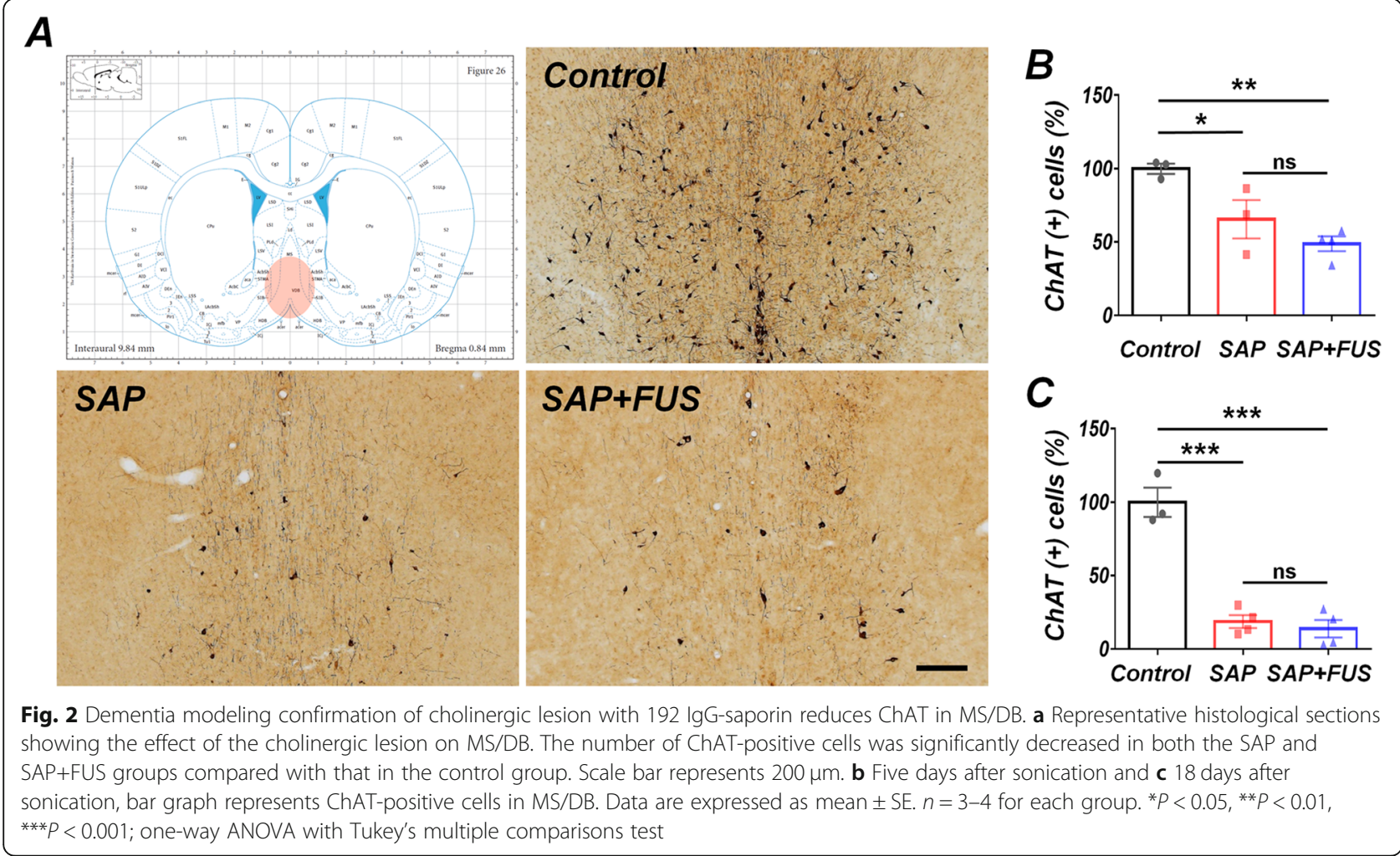




\section{AChE activity at $24 h$ post-sonication}

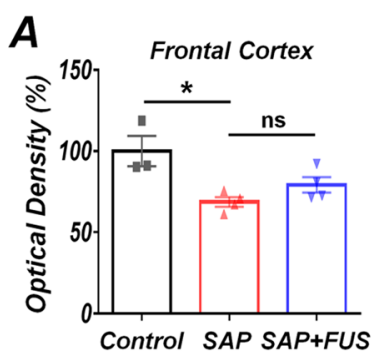

\section{$B$}

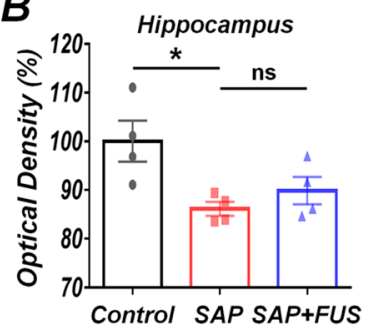

AChE activity at $18 \mathrm{~d}$ post-sonication

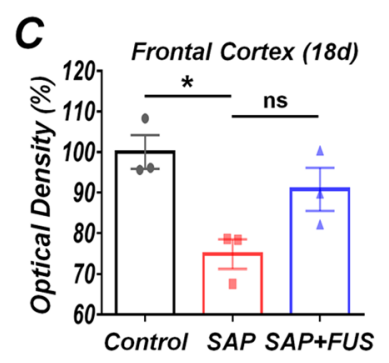

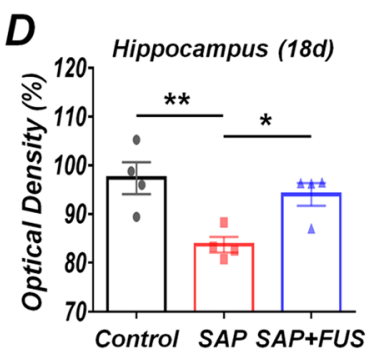

Mature-BDNF expression 24h post-sonication Mature-BDNF expression at 18 d post-sonication

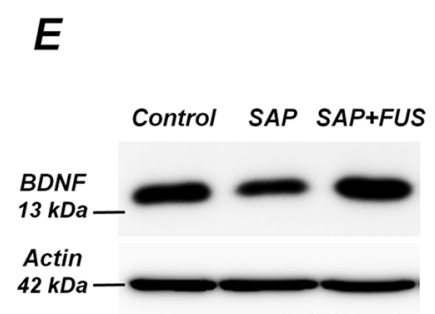
Fig.
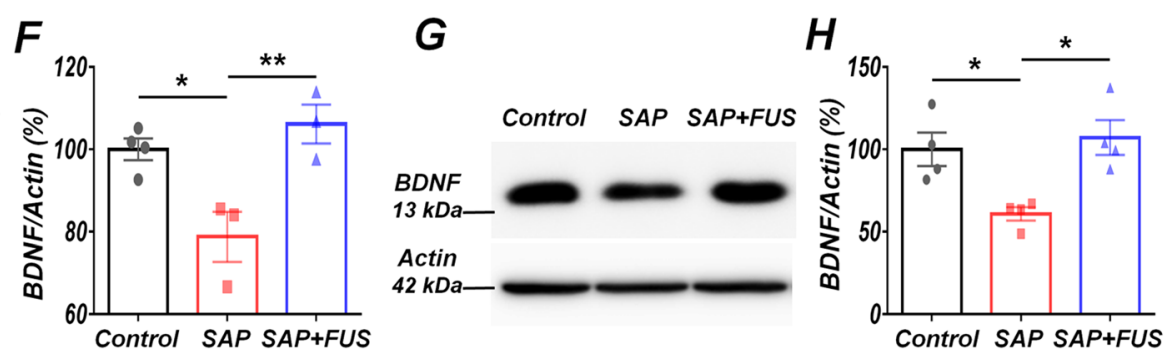

Fig. 3 FUS increases AChE activity BDNF expression levels in a dementia rat model. a Twenty-four hours after sonication, AChE activity was significantly decreased in FC and $\mathbf{b}$ the hippocampus. $\mathbf{c}$ Eighteen days after sonication, FUS-mediated BBB opening induced a significant increase in AChE activity in FC and $\mathbf{d}$ the hippocampus. e Immunoblotting analysis shows BDNF protein expression levels in the hippocampus $24 \mathrm{~h}$ after sonication. BDNF levels in the FUS group increased significantly compared with those in the SAP and control groups. $\mathbf{f}$ Bar graph represents BDNF expression levels in the hippocampus. $\mathbf{g}$ Eighteen days after sonication, BDNF expression in the hippocampus in the FUS group increased significantly compared with that in the SAP and control groups. $\mathbf{h}$ Bar graph represents BDNF expression levels in the hippocampus. Data are expressed as mean \pm SE. $n=3-4$ for each group. ${ }^{*} P<0.05$, ${ }^{* *} P<0.01$; one-way ANOVA with Tukey's multiple comparisons test

AChE activities was only observed in the hippocampus at 18 days.

\section{FUS increases mature-BDNF expression in a dementia rat model}

BDNF acts on specific neurons by promoting neurogenesis, which is crucial for long-term memory. To examine the effects of FUS on BDNF expression in the hippocampus, we performed immunoblotting analyses using brain samples from the hippocampal region obtained at $24 \mathrm{~h}$ and 18 days after sonication. The BDNF gene produces immature BDNF protein $(17 \sim 32 \mathrm{kDa})$ and BDNF mature form $(\sim 13 \mathrm{kDa})$ by intracellular and extracellular proteases (Additional file 1: Figure S2) [39]. At both time points, compared with the control group, the SAP group (24 h: $80.15 \pm 6.16 \%$; 18 days: $60.79 \pm 4.09 \% ; P<0.05)$ exhibited a significantly reduced expression level of mature-BDNF in the hippocampus, whereas compared with the SAP group, the SAP+FUS group (24 h: $108 \pm 4.81 \%$; $P<0.01 ; 18$ days: $73.37 \pm 10.63 \%$; $P<0.05)$ showed a significantly increased level of matureBDNF (Fig. 3e-h).

The cholinergic degeneration of MS induced decreased expression level of BDNF in the hippocampus at $24 \mathrm{~h}$ and 18 days. In contrast, FUS could upregulate the BDNF at the same time points.
FUS affects EGR1 activity in a dementia rat model

EGR1, a transcriptional regulator, is extensively used as a marker for neuronal plasticity. To investigate whether FUS affects the transcription factor of EGR1 expression at 5 days after sonication, the number of EGR1-positive cells was visualized using immunohistochemistry. The SAP group exhibited a significantly lower number of EGR1-positive cells in CA1 $(117 \pm 4 ; P<0.001)$, CA3 $(67 \pm 9 ; P<0.01)$, and DG $(159 \pm 6 ; P<0.01)$ of the hippocampus than the control group (CA1, $163 \pm 2$; CA3, $87 \pm 4$; DG, $229 \pm 15$ ). However, the EGR1 activity in the FUS group indicated a significant increase in CA1 $(135 \pm 4 ; \quad P<0.05), \quad$ CA3 $(77 \pm 4 ; \quad P<0.05)$, and DG $(199 \pm 5 ; P<0.05)$ compared with that in the SAP group (Fig. 4a, b).

These results indicate that cholinergic degeneration of MS caused the decreased activities of EGR1 in hippocampal region at 5 days, and FUS significantly upregulated the activities of EGR1 than SAP group.

\section{Effect of FUS exposure on the proliferation and neuroblast production in DG}

The rats in each group were sacrificed 5 days after bilateral sonication of the hippocampal regions. Twenty-four hours after sonication, BrdU labeling was performed for 


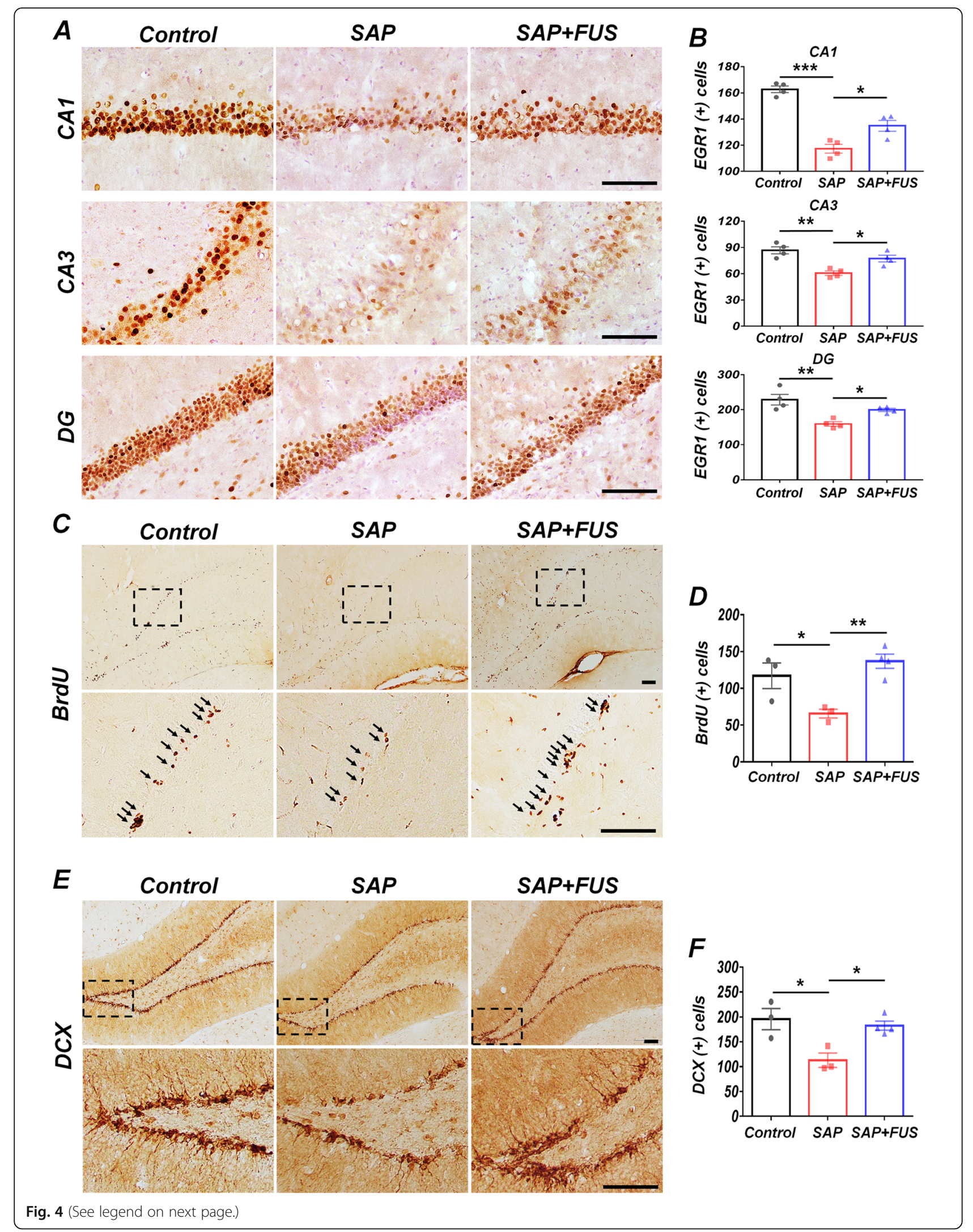


(See figure on previous page.)

Fig. 4 FUS upregulates EGR1 and proliferates neuroblast production in a dementia rat model. a Representative EGR1-stained brain sections 5 days after sonication. Compared with the SAP and control groups, the SAP+FUS group showed a significant increase in EGR1-positive cells in CA1, CA3, and DG of the hippocampus. Scale bar represents $100 \mu \mathrm{m}$. b Quantification of bar graph displays the number of EGR1-positive cells in CA1, CA3, and DG of the hippocampus. $\mathbf{c}$ Representative BrdU-stained brain sections 5 days after sonication. Compared with the SAP and control groups, the SAP+FUS group showed a significant increase in BrdU-positive cells in the SGZ of the DG of the hippocampus. Scale bar represents $100 \mu \mathrm{m}$. d Bar graph displays the number of BrdU-positive cells in SGZ of DG. e Representative DCX-stained brain sections 5 days after sonication. Compared with the SAP and control groups, the SAP+FUS group showed a significant increase in DCX-positive cells in SGZ of DG. Scale bar represents $100 \mu \mathrm{m}$. $\mathbf{f}$ Bar graph displays the number of DCX-positive cells in SGZ of DG. Data are expressed as mean \pm SE. $n=3-4$ for each group. ${ }^{*} P<0.05,{ }^{*} P<0.01,{ }^{* * *} P<0.001$; one-way ANOVA with Tukey's multiple comparisons test. The scale bar represents $100 \mu \mathrm{m}$

4 consecutive days for each group to observe progenitor cell proliferation in SGZ of DGs. We observed a decrease in the number of BrdU-positive cells in the SAP group $(65 \pm 6 ; P<0.05)$ compared with that in the control group $(117 \pm 18)$, while this number was significantly increased in the SAP+FUS group $(137 \pm 10 ; P<0.01)$ compared with that in the SAP group (Fig. 4c, d).

To investigate whether FUS affects newly generated immature neurons, the number of neuroblasts was visualized using doublecortin (DCX, a marker for neurogenesis) immunohistochemistry. Compared with the control $(196 \pm 21 ; P<0.05)$ and SAP+FUS $(183 \pm 9 ; \quad P<0.05$; Fig. 4e, f) groups, the SAP group exhibited a significantly reduced number of DCX-positive cells in DG of the hippocampus (113 \pm 14$)$.

It has been suggested that cholinergic degeneration of MS showed decreased activities of proliferation and neuroblast production in SGZ at $24 \mathrm{~h}$ and 18 days, which was reversed by FUS.

\section{FUS affects neurogenesis in a dementia rat model}

To determine the phenotypic characterization of BrdUpositive cells 18 days after sonication, sections were analyzed 2 weeks after the last injection of BrdU: neuronal phenotypes were identified by double-immunofluorescence labeling for NeuN and BrdU and glial phenotypes by double-immunofluorescence labeling for GFAP (astrocytespecific marker) and BrdU (Fig. 5a-c). Compared with the control group ( $40 \pm 2)$, the SAP group $(25 \pm 2$; $P<0.01)$ displayed significantly reduced neurogenesis $(\mathrm{NeuN}+/ \mathrm{BrdU}+)$ in SGZ/GCL of DG. Compared with the SAP group, the SAP+FUS group $(49 \pm 1 ; P<0.001)$ featured a significantly increased number of co-expression cells $(\mathrm{NeuN}+/ \mathrm{BrdU}+)$ in DG. No significant differences in gliogenesis (GFAP+/ BrdU+) (Fig. 5e) and the phenotypes of BrdU-positive cells expressing NeuN or GFAP in DG (Fig. 5g) were identified among the groups.

The decreased AHN induced by cholinergic degeneration of MS was enhanced by FUS at 18 days. Interestingly, gliogenesis was not affected by FUS.

\section{FUS improved performance in the MWM task}

To investigate the effects of FUS on memory and cognitive function, rats ( $n=8$ /group) underwent training for
MWM for 5 consecutive days (14 days post-modeling). All groups showed a gradual decrease in escape latency to the platform from the first day to the last day of the training (Fig. 6a).

In the MWM probe test, rats in the SAP group made significantly a lower number of crossing over $(0.62 \pm$ $0.26 ; P<0.01)$ and spent less time in the platform area $(0.47 \pm 0.16 ; P<0.05)$ compared with those in the control group (crossing over, $3.37 \pm 0.7$; platform area, $1.44 \pm 0.32$ ). In contrast, rats in the SAP+FUS group showed a significantly higher number of crossing over $(3.12 \pm 0.61 ; P<0.05)$ and time in the platform area $(1.51 \pm 0.21 ; P<0.05)$ compared with those in the control group (Fig. 6b, c). However, the time spent in the target quadrant and movement speed were not significantly different among the groups (Fig. 6d, e).

These results implicate that cholinergic degradation in MS reduces the memory and cognitive function, but FUS can reverse the impairment.

\section{Discussion}

Although FUS may remedy the impermeability of BBB to pharmacotherapy, the effects of FUS on cholinergic function and AHN have not been elucidated in cholinergic-deficient conditions. Cholinergic systems regulate memory processing and cognitive function and link the memory circuit constituted by FC, hippocampus, and MS [40, 41]. The present study was designed to measure and analyze the potential effects of FUS in a rat model of dementia mimicking the BFC depletion in AD. The cholinergic dysfunction in this model was induced by intraventricular injection of SAP. This immunotoxin acts by coupling the ribosome-inactivating protein to a monoclonal antibody, which has a low affinity for the nerve growth factor receptor p75, located on BFC neuron bodies [42, 43]. In this study, we examined the cholinergic degeneration in this model, and our results revealed that the number of ChAT neurons was significantly reduced in MS of the SAP and SAP+FUS groups both 5 days and 18 days after FUS (Fig. 2), which proves that cholinergic dysfunction was well-established in this rat model, and the results are consistent with our previous results [33-36, 44, 45]. 

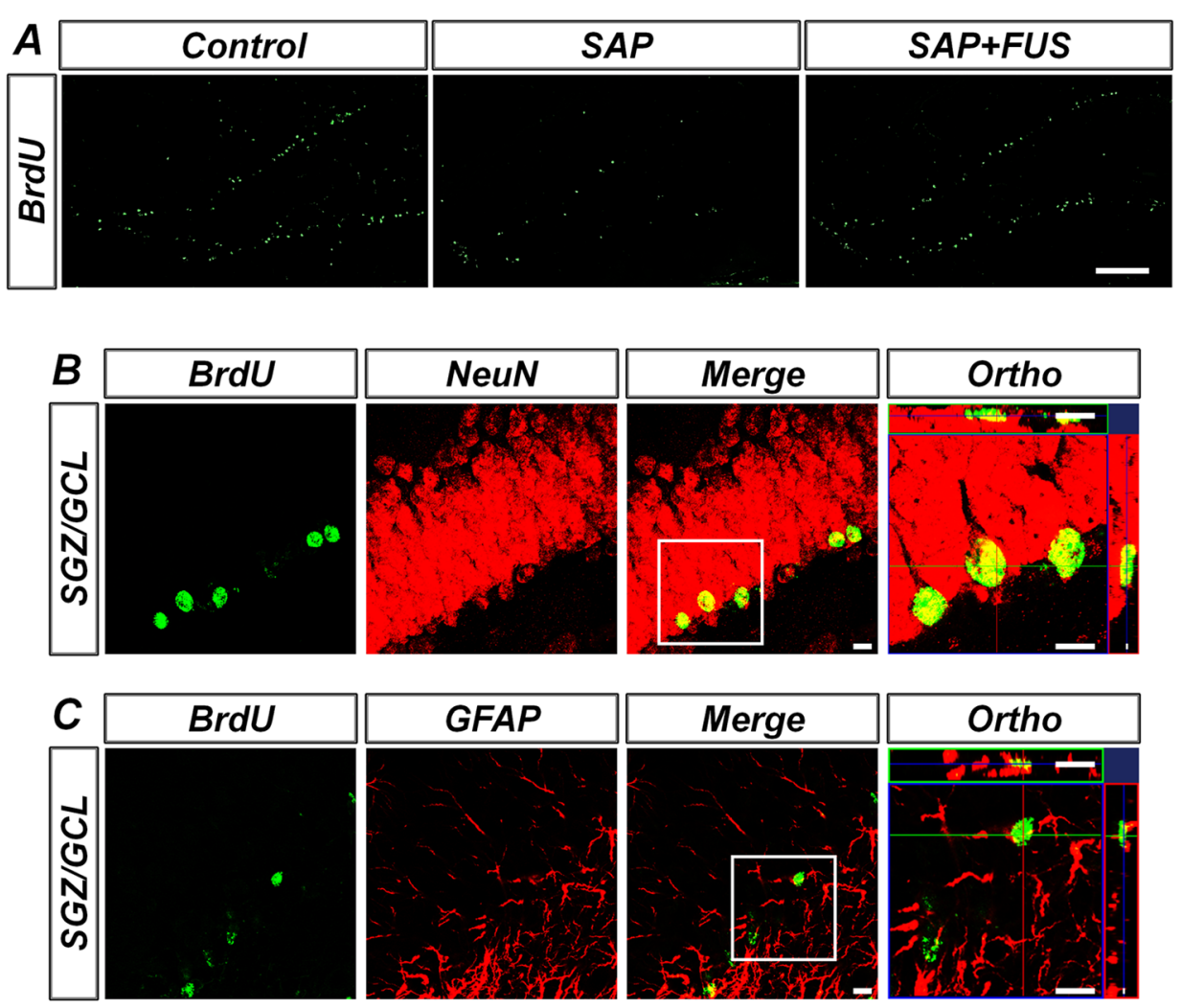

$D$

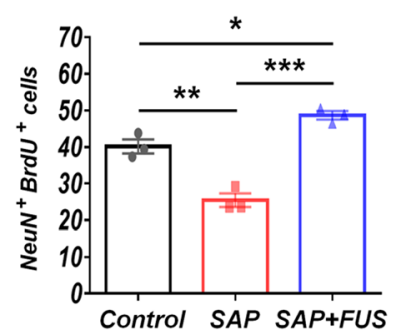

$E$

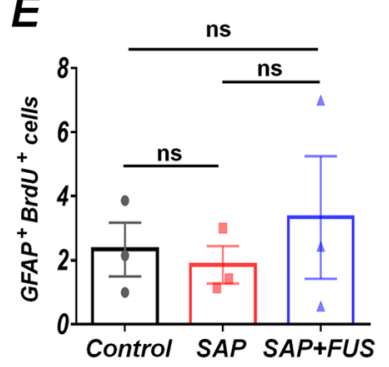

$F$

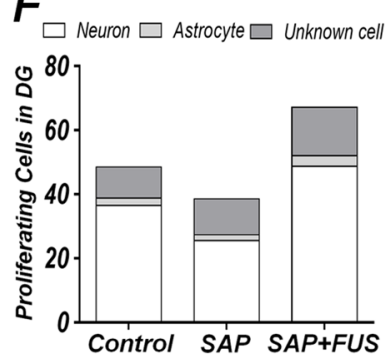

G

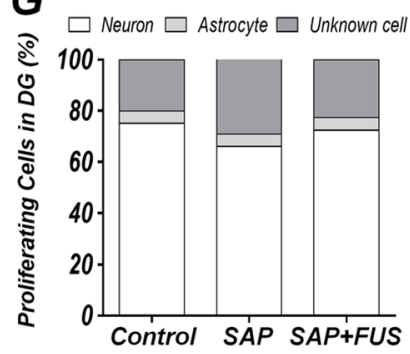

Fig. 5 FUS increases neurogenesis and does not affect gliogenesis in a dementia rat model. a Representative photographs show the distribution of survived proliferation of BrdU-labeled positive cells in SGZ/GCL of DG of the hippocampus 18 days after sonication. The scale bar represents $200 \mu \mathrm{m}$. b Representative photographs of BrdU (green, a proliferative cell marker) and NeuN (red, a neuron marker) and c BrdU (green, a proliferative cell marker) and GFAP (red, an astrocyte marker) double-labeled cells in SGZ/GCL of DG of the hippocampus at 18 days after sonication. The scale bar represents $20 \mu \mathrm{m}$. d Quantification of BrdU and NeuN double-labeled cells. Compared with the SAP and control groups, the SAP+FUS group showed a significant increase in BrdU/NeuN-positive cells. e No significant differences were found in the numbers of BrdU/ GFAP-positive cells among the groups. $\mathbf{f}$ Survived newly generating cells were determined, indicative of neurogenesis and gliogenesis. $\mathbf{g}$ The overall proportion of cells with the SGZ/GCL phenotype in DG of the hippocampus among the groups. Data are expressed as mean \pm SE. $n=3-4$ for each group. ${ }^{*} P<0.05,{ }^{*} P<0.01$; one-way ANOVA with Tukey's multiple comparisons test

Because the reduction in AChE activity by cholinergic lesions in the hippocampus is closely correlated with Ach and AChE following compensatory mechanisms induced by SAP, we observed AChE activity in FC and the hippocampus [46] to examine the effects of FUS. Interestingly, the FUS group also exhibited decreases in the activity at $24 \mathrm{~h}$, suggesting that sonication did not acutely affect AChE activity at $24 \mathrm{~h}$ (Fig. 3a, b). However, the SAP+FUS group showed a significantly increased
AChE activity in the hippocampus 18 days after sonication (Fig. 3c, d), which implies that the FUS-mediated $\mathrm{BBB}$ opening resulted in the recovery of AChE levels.

EGR1 can activate AChE gene expression by binding to the AChE promoter. Overexpression of acetylcholine and $\mathrm{AChE}$ is critical for upregulating proliferative activity and subsequent neurogenesis [46]. Furthermore, acetylcholine modulates hippocampal long-term potentiation (LTP), thereby stimulating cholinergic neurons 


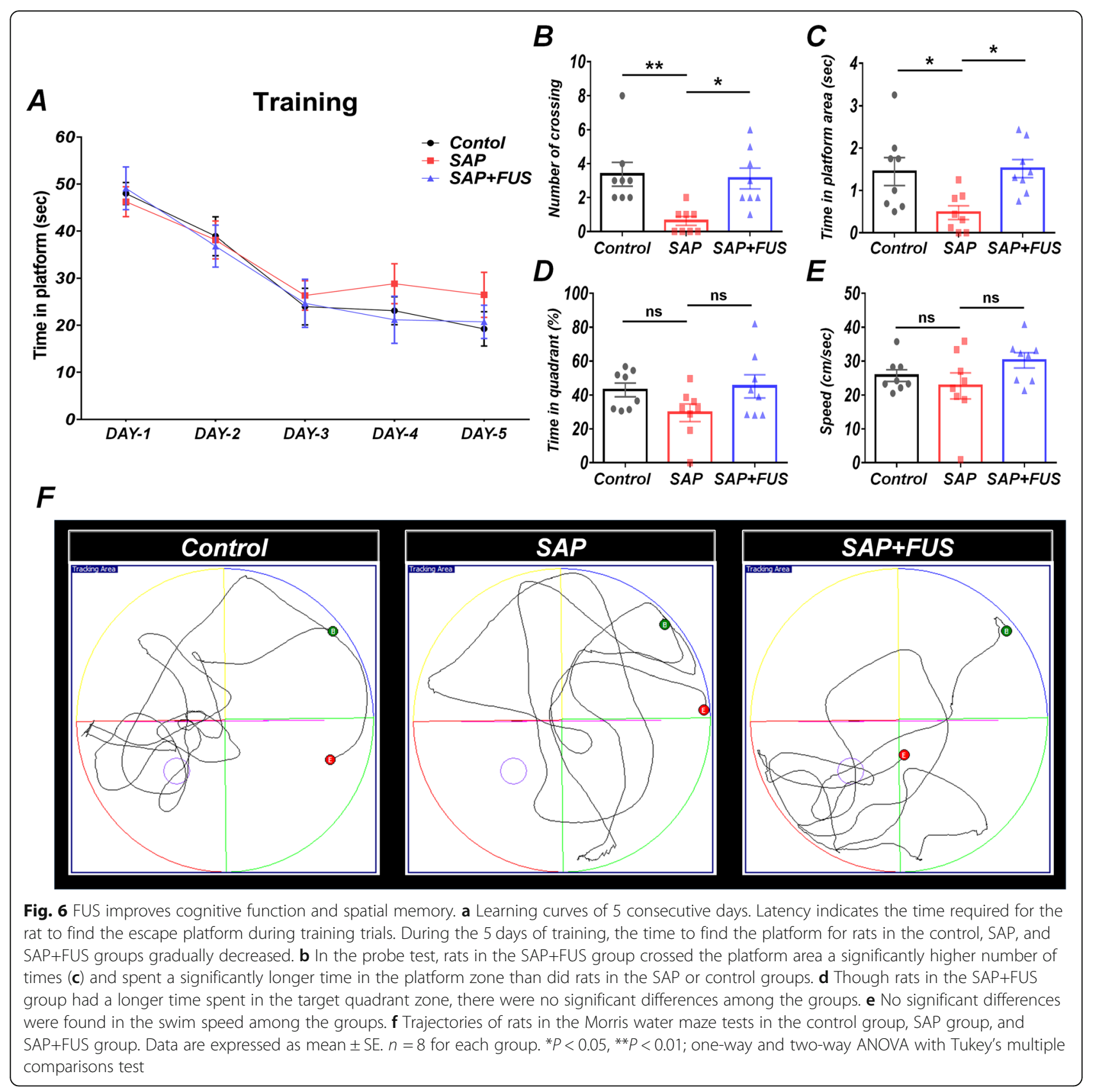

and enhancing hippocampal LTP $[47,48]$. Another factor contributing to neurogenesis is BDNF, which has gained attention for its role in the regulation of synaptic transmission and plasticity, and neural circuit function in the CNS [49]. An insufficient supply of endogenous BDNF leads to neurodegeneration, cognitive impairment, and sharp decreases in neuronal proliferation in SGZ [50, 51]. Furthermore, dysfunction in the cholinergic forebrain system diminishes AHN in DG [52]. FUS increases neurogenesis in wild-type mice [29], and the effect of BDNF endures even $24 \mathrm{~h}$ after FUS treatment [24]. Because the correlation between BDNF and AHN has been already proven in previous research [53], our goal was to observe changes in BDNF and EGR1 activities in this model and how those factors could recover via FUS.

Consistent with previous observations, we demonstrated that the cholinergic-deficient conditions in the SAP group significantly reduced BDNF expression levels in the hippocampus (Fig. 3e, f) [54, 55]. The present study further demonstrated that the FUS-mediated BBB opening elevated BDNF expression both $24 \mathrm{~h}$ and 18 days after sonication of the hippocampus (Fig. $3 \mathrm{f}-\mathrm{h}$ ) and improved neurogenesis in GCL/SGZ of DG (Fig. 5d). 


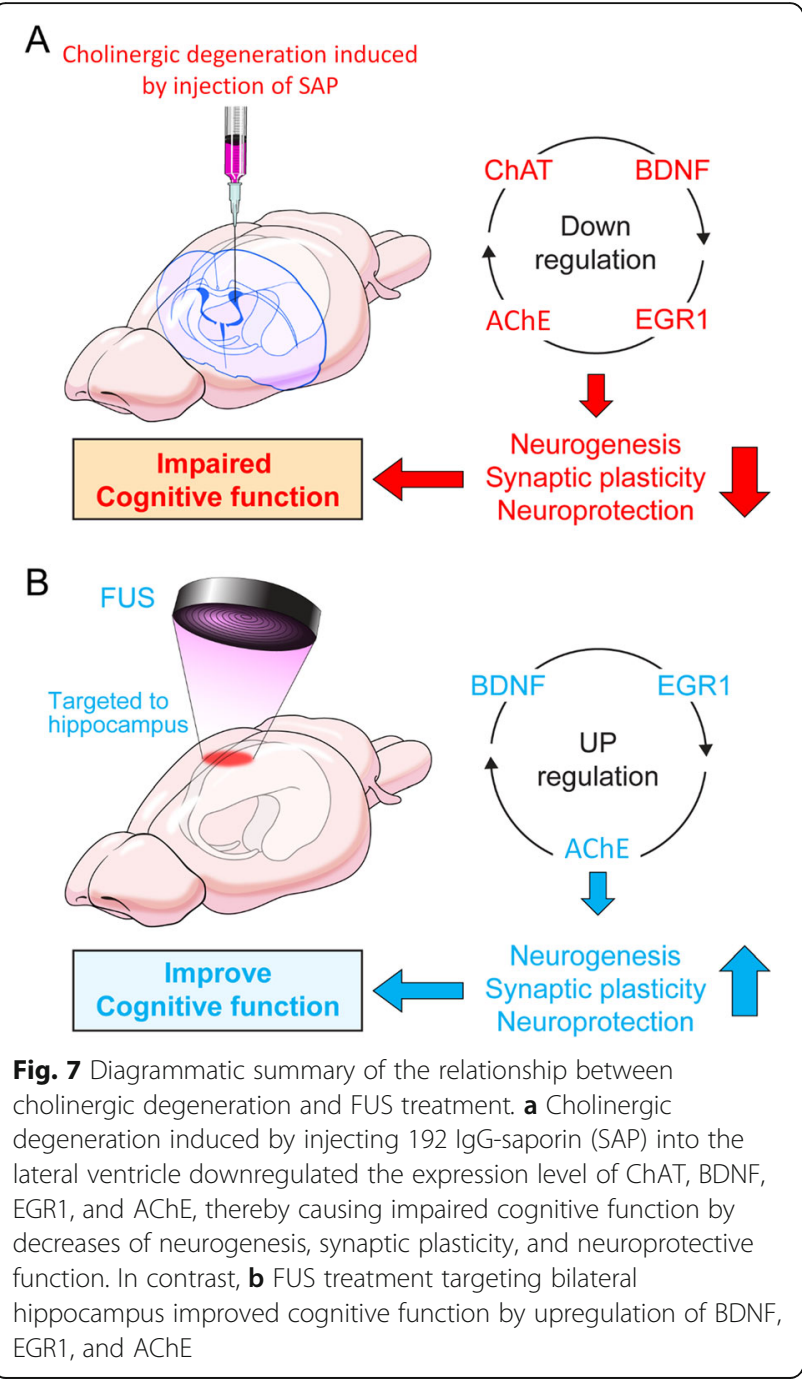

These results indicate that FUS can promote BDNF expression $24 \mathrm{~h}$ after sonication, thereby confirming the results of previous research [24]. The mature BDNF primarily binds to the $\operatorname{TrkB}$ receptor, which plays a role in the development, maintenance, and differentiation of neurons and cell survival [56-58]. The maintenance of elevated expression of BDNF 18 days after sonication suggests that BDNF continuously regulates neurogenesis, synaptic plasticity, and membrane excitability $[59,60]$. Our results indicate that diminished cholinergic input to the hippocampus reduces BDNF expression; FUSmediated opening of $\mathrm{BBB}$ reverses these effects by stimulating hippocampal BDNF expression, which consequently regulates AHN positively in cholinergic degeneration [4].

Compared with the control group, the SAP group displayed decreased levels of EGR1 in the hippocampus (Fig. 4a), whereas EGR1 activity in the FUS group was significantly increased compared with that in the SAP group. Prior evidence has demonstrated that extracellular BDNF activates ERK expression by TrkB neurotrophin receptor [61]. The activation of transcription factors, such as CREB and IEGs including c-fos and Egr1, is followed by increased ERK phosphorylation [62]. This activation may play a critical role in BDNF upregulation induced by FUS, which could potentially contribute to the upregulation of EGR1 (Fig. 4a). In many studies, EGR1 transcription factors have been demonstrated to be major regulators and mediators of synaptic activity and plasticity under certain physiological conditions [63, 64]. Thus, our findings support prior evidence that BDNF facilitates the return of EGR1 to normal levels.

Our data support the theory that forebrain acetylcholine affects $\mathrm{AHN}$, and a selective cholinergic lesion of the BFC system induces a decrease in BrdU, EGR1, $\mathrm{DCX}$, and $\mathrm{AChE}$ levels; therefore, these findings indicate a reduction in proliferation and neuroblast production in SGZ and a decrease in hippocampal acetylcholine activity, respectively [35]. We found that the FUSmediated BBB opening led to an increase in BDNF, EGR1, and AHN levels, which lead to an improvement in cognitive function.

Based on results from the behavioral test, we could also confirm that FUS enhanced memory and cognitive function. The performance of all rats in all groups gradually improved across 5 days of MWM training, suggesting that rats with cholinergic dysfunction have a similar level of learning capacity and escape latency compared with wild-type rats (Fig. 6) [34, 35]. In the probe test, when compared with the control and FUS groups, the SAP group displayed a diminished MWM performance $72 \mathrm{~h}$ after final training, as measured by the number of crossing over the platform area and time spent, which complements the findings of previous studies [33-36, $44]$. However, FUS improved spatial memory, and cognition correlated with increases in EGR1, BDNF, and AHN. According to a recent study, increases in both AHN and BDNF levels affected memory improvement, similar to the effects of exercise in $A D$ transgenic mouse [65]. However, increases in AHN alone did not have any effect [65]. Significant differences in speed were not observed among the groups, suggesting that there are no SAP-induced differences in motor function (Fig. 6e), which is consistent with our previous findings [35].

Although the data herein showed remarkable effects of FUS in this rat model, this study has several limitations that should be addressed in future research. We fixed sonication parameters to induce $0.25 \mathrm{MPa}$ of acoustic pressure, which was adopted from our previous study [66]. However, recent studies have used an acoustic feedback system based on the passive cavitation detector to prevent tissue damage. This technique may guarantee appropriate sonication power and could be suitable for 
clinical application. Our previous study demonstrated that a FUS-mediated BBB opening could be safely and effectively performed within certain parameters [66]. Moreover, we used MRI to confirm BBB opening (T1W) without cell edema (T2W) after sonication (Fig. 1e, f). Furthermore, we observed increased BDNF expression only at $24 \mathrm{~h}$ and 18 days after sonication so different time points between $24 \mathrm{~h}$ and 18 days could be further studied to assess the changes in BDNF. In addition, we observed the recovery effects of FUS on EGR1 activities at 5 days in the model; thus, based on these results, we could assume that neuroblast production and cell migration might have maintained [53]. We could anticipate LTP and synaptic strength would recover.

\section{Conclusions}

In the present study, we demonstrated that animals with BFC hypofunction causing spatial memory impairment exhibit a reduction in cholinergic activity, neurogenesis, and BDNF and ERG1 expression levels (Fig. 7a). In contrast, FUS treatment increased $\mathrm{AHN}$ and improved spatial memory in cholinergic degeneration conditions. This improvement may be mediated by the upregulation of BDNF, EGR1, and AChE levels in the hippocampus, which is a critical factor for regulating AHN, synaptic plasticity, and neuroprotection (Fig. 7b). Because patients with $\mathrm{AD}$ have impaired cholinergic neurons and AHN starting at the early stages, FUS treatment may restore AHN and have a protective effect against neurodegeneration. Moreover, as FUS has been shown to be effective in increasing $\mathrm{AHN}$, it could also contribute to increased permeability of $\mathrm{BBB}$ for drug delivery, and both these effects could be potential therapeutic strategies for AD.

\section{Supplementary information}

Supplementary information accompanies this paper at https://doi.org/10. 1186/s13195-019-0569-x

Additional file 1: Figure S1. The schematic of the FUS experimental setup. Figure S2. Immunoblot of BDNF of multiple bands at matureBDNF ( $13 \mathrm{kDa})$ and immature-BDNF (17 32 kDa) were observed at (A) twenty-four hours after sonication and (B) eighteen days after sonication.

\section{Abbreviations}

AHN: Adult hippocampal neurogenesis; AChE: Acetylcholinesterase; AD: Alzheimer's disease; ANOVA: Analysis of variance; BBB: Blood-brain barrier; BDNF: Brain-derived neurotrophic factor; BFC: Basal forebrain cholinergic; ChAT: Choline acetyltransferase; CNS: Central nervous system; DCX: Doublecortin; DG: Dentate gyrus; FC: Frontal cortex; GCL: Granular cell layer; FUS: Focused ultrasound; MRI: Magnetic resonance imaging; MS/ DB: Medial septum-diagonal band complex; MWM: Morris water maze; PBS: Phosphate-buffered saline; SAP: 192 IgG-saporin; SGZ: Subgranular zone

\section{Acknowledgements}

The authors would like to thank Dong-Su Jang, MFA (Medical Illustrator, Medical Research Support Section, Yonsei University College of Medicine, Seoul, Korea), for his help with our illustrations.

\section{Authors' contributions}

JWC, WSC, and JS designed the experiments. CK, JS (Jiyeon Sim), JL, and JS performed the experiments. BYC, SWS, CK, and IS analyzed the data. JWC, WSC, YCN, and JS wrote the paper. WSC, BYC, MP, CS, and JS revised the manuscript. All authors contributed to the discussion. All authors read and approved the final manuscript.

\section{Funding}

This study was supported by grants from the Brain Research Program through the National Research Foundation of Korea (NRF) funded by the Korean Ministry of Science, ICT, and Future Planning (2016M3C7A1914123) and a grant of the Korea Health Technology R\&D Project through the Korea Health Industry Development Institute (KHIDI), funded by the Ministry of Health \& Welfare, Republic of Korea (grant number: HI19C0060). The funding source had no involvement in the study design, data collection, analysis, and interpretation, in the writing of the report, or in the decision to submit the article for publication.

\section{Availability of data and materials}

The datasets used and/or analyzed during the current study are available from the corresponding author on reasonable request.

\section{Ethics approval and consent to participate}

All animal experimental procedures were conducted in compliance with the Guide for the Care and Use of Laboratory Animals of the National Institutes of Health and were approved by the Institutional Animal Care and Use Committee (IACUC; 2016-0339) of Yonsei University.

\section{Consent for publication}

Not applicable.

\section{Competing interests}

The authors declare that they have no competing interests.

\section{Author details}

'Department of Neurosurgery, Yonsei University College of Medicine, Seoul 03722, Republic of Korea. ${ }^{2}$ Brain Korea 21 PLUS Project for Medical Science and Brain Research Institute, Yonsei University College of Medicine, Seoul 03722, Republic of Korea. ${ }^{3}$ Department of Physiology, Hallym University College of Medicine, Chuncheon 24252, Republic of Korea. ${ }^{4}$ Department of Neurosurgery, Catholic Kwandong University College of Medicine, International St Mary's Hospital, Incheon Metropolitan City 22771, Republic of Korea.

Received: 10 July 2019 Accepted: 12 December 2019

Published online: 27 December 2019

\section{References}

1. Altman J, Das GD. Autoradiographic and histological evidence of postnatal hippocampal neurogenesis in rats. J Comp Neurol. 1965;124(3):319-35.

2. Eriksson PS, Perfilieva E, Bjork-Eriksson T, Alborn AM, Nordborg C, Peterson DA, et al. Neurogenesis in the adult human hippocampus. Nat Med. 1998; 4(11):1313-7.

3. Ming G-L, Song $\mathrm{H}$. Adult neurogenesis in the mammalian brain: significant answers and significant questions. Neuron. 2011;70(4):687-702.

4. Zhao C, Deng W, Gage FH. Mechanisms and functional implications of adult neurogenesis. Cell. 2008;132(4):645-60.

5. Tobin MK, Musaraca K, Disouky A, Shetti A, Bheri A, Honer WG, et al. Human hippocampal neurogenesis persists in aged adults and Alzheimer's disease patients. Cell Stem Cell. 2019;24(6):974-82.e3.

6. Boldrini M, Fulmore CA, Tartt AN, Simeon LR, Pavlova I, Poposka V, et al. Human hippocampal neurogenesis persists throughout aging. Cell Stem Cell. 2018;22(4):589-99.e5.

7. Scheltens P, Blennow K, Breteler MM, de Strooper B, Frisoni GB, Salloway S, et al. Alzheimer's disease. Lancet. 2016;388(10043):505-17.

8. Ginsberg SD, Che S, Wuu J, Counts SE, Mufson EJ. Down regulation of trk but not P75NTR gene expression in single cholinergic basal forebrain neurons mark the progression of Alzheimer's disease. J Neurochem. 2006; 97(2):475-87. 
9. Grothe M, Heinsen H, Teipel SJ. Atrophy of the cholinergic basal forebrain over the adult age range and in early stages of Alzheimer's disease. Biol Psychiatry. 2012;71(9):805-13.

10. Pappas BA, Bayley PJ, Bui BK, Hansen LA, Thal L. Choline acetyltransferase activity and cognitive domain scores of Alzheimer's patients 2 . Neurobiol Aging. 2000;21(1):11-7.

11. Manseau F, Danik M, Williams S. A functional glutamatergic neurone network in the medial septum and diagonal band area. J Physiol. 2005; 566(Pt 3):865-84.

12. Baxter MG, Chiba AA. Cognitive functions of the basal forebrain. Curr Opin Neurobiol. 1999;9(2):178-83.

13. Perry EK, Tomlinson BE, Blessed G, Bergmann $\mathrm{K}$, Gibson PH, Perry RH. Correlation of cholinergic abnormalities with senile plaques and mental test scores in senile dementia. Br Med J. 1978;2(6150):1457-9.

14. Bartus RT. On neurodegenerative diseases, models, and treatment strategies: lessons learned and lessons forgotten a generation following the cholinergic hypothesis. Exp Neurol. 2000;163(2):495-529.

15. Coyle JT, Price DL, Delong MR. Alzheimer's disease: a disorder of cortical cholinergic innervation. Science. 1983;219(4589):1184-90.

16. Ricceri L, Minghetti L, Moles A, Popoli P, Confaloni A, De Simone R, et al. Cognitive and neurological deficits induced by early and prolonged basal forebrain cholinergic hypofunction in rats. Exp Neurol. 2004;189(1):162-72.

17. Cummings JL, Morstorf T, Zhong K. Alzheimer's disease drug-development pipeline: few candidates, frequent failures. Alzheimers Res Ther. 2014;6(4):37.

18. Pardridge WM. Blood-brain barrier drug targeting: the future of brain drug development. Mol Interv. 2003;3(2):90.

19. Park J, Aryal M, Vykhodtseva N, Zhang Y-Z, McDannold N. Evaluation of permeability, doxorubicin delivery, and drug retention in a rat brain tumor model after ultrasound-induced blood-tumor barrier disruption. J Control Release. 2017;250:77-85

20. Fan C-H, Lin C-Y, Liu H-L, Yeh C-K. Ultrasound targeted CNS gene delivery for Parkinson's disease treatment. J Control Release. 2017;261:246-62.

21. Zhu L, Zhao H, Zhou Z, Xia Y, Wang Z, Ran H, et al. Peptide-functionalized phase-transformation nanoparticles for low intensity focused ultrasoundassisted tumor imaging and therapy. Nano Lett. 2018;18(3):1831-41.

22. Zlokovic BV. The blood-brain barrier in health and chronic neurodegenerative disorders. Neuron. 2008;57(2):178-201.

23. Yoo SS, Bystritsky A, Lee JH, Zhang Y, Fischer K, Min BK, et al. Focused ultrasound modulates region-specific brain activity. Neuroimage. 2011;56(3): 1267-75.

24. Kovacs ZI, Kim S, Jikaria N, Qureshi F, Milo B, Lewis BK, et al. Disrupting the blood-brain barrier by focused ultrasound induces sterile inflammation. Proc Natl Acad Sci U S A. 2017;114(1):E75-e84.

25. Tufail Y, Matyushov A, Baldwin N, Tauchmann ML, Georges J, Yoshihiro A, et al. Transcranial pulsed ultrasound stimulates intact brain circuits. Neuron. 2010;66(5):681-94.

26. Burgess A, Dubey S, Yeung S, Hough O, Eterman N, Aubert I, et al. Alzheimer disease in a mouse model: MR imaging-guided focused ultrasound targeted to the hippocampus opens the blood-brain barrier and improves pathologic abnormalities and behavior. Radiology. 2014;273(3): $736-45$.

27. Jordao JF, Thevenot E, Markham-Coultes K, Scarcelli T, Weng YQ, Xhima K, et al. Amyloid-beta plaque reduction, endogenous antibody delivery and glial activation by brain-targeted, transcranial focused ultrasound. Exp Neurol. 2013;248:16-29.

28. Leinenga G, Gotz J. Scanning ultrasound removes amyloid-beta and restores memory in an Alzheimer's disease mouse model. Sci Transl Med. 2015;7(278):278ra33.

29. Scarcelli T, Jordao JF, O'Reilly MA, Ellens N, Hynynen K, Aubert I. Stimulation of hippocampal neurogenesis by transcranial focused ultrasound and microbubbles in adult mice. Brain Stimul. 2014;7(2):304-7.

30. Mooney SJ, Shah K, Yeung S, Burgess A, Aubert I, Hynynen K. Focused ultrasound-induced neurogenesis requires an increase in blood-brain barrier permeability. PLoS One. 2016;11(7):e0159892.

31. Moreno-Jiménez EP, Flor-García M, Terreros-Roncal J, Rábano A, Cafini F, PallasBazarra N, et al. Adult hippocampal neurogenesis is abundant in neurologically healthy subjects and drops sharply in patients with Alzheimer's disease. Nat Med. 2019. https://doi.org/10.1038/s41591-019-0375-9.

32. Wiley RG, Oeltmann TN, Lappi DA. Immunolesioning: selective destruction of neurons using immunotoxin to rat NGF receptor. Brain Res. 1991;562(1): 149-53.
33. Lee JE, Jeong da U, Lee J, Chang WS, Chang JW. The effect of nucleus basalis magnocellularis deep brain stimulation on memory function in a rat model of dementia. BMC Neurol. 2016;16:6.

34. Cho JS, Lee J, Jeong DU, Kim HW, Chang WS, Moon J, et al. Effect of placenta-derived mesenchymal stem cells in a dementia rat model via microglial mediation: a comparison between stem cell transplant methods. Yonsei Med J. 2018;59(3):406-15.

35. Jeong DU, Lee J, Chang WS, Chang JW. Identifying the appropriate time for deep brain stimulation to achieve spatial memory improvement on the Morris water maze. BMC Neurosci. 2017;18(1):29.

36. Jeong DU, Chang WS, Hwang YS, Lee D, Chang JW. Decrease of GABAergic markers and arc protein expression in the frontal cortex by intraventricular 192 lgG-saporin. Dement Geriatr Cogn Disord. 2011;32(1):70-8.

37. Choi BY, Kim IY, Kim JH, Lee BE, Lee SH, Kho AR, et al. Administration of zinc plus cyclo-(His-Pro) increases hippocampal neurogenesis in rats during the early phase of streptozotocin-induced diabetes. Int J Mol Sci. 2017;18(1). https://www.mdpi.com/1422-0067/18/1/73.

38. Ellman GL, Courtney KD, Andres V Jr, Feather-Stone RM. A new and rapid colorimetric determination of acetylcholinesterase activity. Biochem Pharmacol. 1961;7:88-95.

39. Yang B, Ren Q, JC Z, Chen QX, Hashimoto K. Altered expression of BDNF, BDNF pro-peptide and their precursor proBDNF in brain and liver tissues from psychiatric disorders: rethinking the brain-liver axis. Transl Psychiatry. 2017;7(5):e1128-e.

40. Jerusalinsky D, Kornisiuk E, Izquierdo I. Cholinergic neurotransmission and synaptic plasticity concerning memory processing. Neurochem Res. 1997; 22(4):507-15.

41. Hasselmo ME. The role of acetylcholine in learning and memory. Curr Opin Neurobiol. 2006;16(6):710-5.

42. Book AA, Wiley RG, Schweitzer JB. Specificity of 192 lgG-saporin for NGF receptor-positive cholinergic basal forebrain neurons in the rat. Brain Res. 1992:590(1-2):350-5.

43. Heckers S, Ohtake T, Wiley RG, Lappi DA, Geula C, Mesulam MM. Complete and selective cholinergic denervation of rat neocortex and hippocampus but not amygdala by an immunotoxin against the p75 NGF receptor. J Neurosci. 1994;14(3 Pt 1):1271-89.

44. Jeong DU, Lee JE, Lee SE, Chang WS, Kim SJ, Chang JW. Improvements in memory after medial septum stimulation are associated with changes in hippocampal cholinergic activity and neurogenesis. Biomed Res Int. 2014; 2014:568587.

45. Jeong da U, Oh JH, Lee JE, Lee J, Cho ZH, Chang JW, et al. Basal forebrain cholinergic deficits reduce glucose metabolism and function of cholinergic and GABAergic systems in the cingulate cortex. Yonsei Med J. 2016;57(1): 165-72.

46. Cohen JE, Zimmerman G, Melamed-Book N, Friedman A, Dori A, Soreq H. Transgenic inactivation of acetylcholinesterase impairs homeostasis in mouse hippocampal granule cells. Hippocampus. 2008;18(2):182-92.

47. Blitzer RD, Gil O, Landau EM. Cholinergic stimulation enhances long-term potentiation in the CA1 region of rat hippocampus. Neurosci Lett. 1990; 119(2):207-10

48. Shimoshige Y, Maeda T, Kaneko S, Akaike A, Satoh M. Involvement of M2 receptor in an enhancement of long-term potentiation by carbachol in Schaffer collateral-CA1 synapses of hippocampal slices. Neurosci Res. 1997; 27(2):175-80.

49. Monteggia LM, Barrot M, Powell CM, Berton O, Galanis V, Gemelli T, et al. Essential role of brain-derived neurotrophic factor in adult hippocampal function. Proc Natl Acad Sci. 2004;101(29):10827-32.

50. Taliaz D, Stall N, Dar DE, Zangen A. Knockdown of brain-derived neurotrophic factor in specific brain sites precipitates behaviors associated with depression and reduces neurogenesis. Mol Psychiatry. 2009;15:80.

51. Zuccato C, Cattaneo E. Brain-derived neurotrophic factor in neurodegenerative diseases. Nat Rev Neurol. 2009;5(6):311.

52. Cooper-Kuhn CM, Winkler J, Kuhn HG. Decreased neurogenesis after cholinergic forebrain lesion in the adult rat. J Neurosci Res. 2004;77(2):155-65.

53. Rossi C, Angelucci A, Costantin L, Braschi C, Mazzantini M, Babbini F, et al. Brain-derived neurotrophic factor (BDNF) is required for the enhancement of hippocampal neurogenesis following environmental enrichment. Eur J Neurosci. 2006;24(7):1850-6.

54. Wrenn CC, Wiley RG. The behavioral functions of the cholinergic basal forebrain: lessons from 192 lgG-saporin. Int J Dev Neurosci. 1998;16(7-8): 595-602. 
55. Gil-Bea FJ, Solas M, Mateos L, Winblad B, Ramirez MJ, Cedazo-Minguez A. Cholinergic hypofunction impairs memory acquisition possibly through hippocampal Arc and BDNF downregulation. Hippocampus. 2011;21(9):9991009.

56. Waterhouse EG, An JJ, Orefice LL, Baydyuk M, Liao G-Y, Zheng K, et al. BDNF promotes differentiation and maturation of adult-born neurons through GABAergic transmission. J Neurosci. 2012;32(41):14318-30.

57. Mousavi $\mathrm{K}$, Jasmin BJ. BDNF is expressed in skeletal muscle satellite cells and inhibits myogenic differentiation. J Neurosci. 2006;26(21):5739-49.

58. Reichardt LF. Neurotrophin-regulated signalling pathways. Philos Trans R Soc London B: Biol Sci. 2006;361(1473):1545-64.

59. Bramham CR, Messaoudi E. BDNF function in adult synaptic plasticity: the synaptic consolidation hypothesis. Prog Neurobiol. 2005;76(2):99-125.

60. Scharfman H, Goodman J, Macleod A, Phani S, Antonelli C, Croll S. Increased neurogenesis and the ectopic granule cells after intrahippocampal BDNF infusion in adult rats. Exp Neurol. 2005;192(2):348-56.

61. M-m P. Neurotrophins as synaptic modulators. Nat Rev Neurosci. 2001;2(1): 24.

62. Impey S, Obrietan K, Wong ST, Poser S, Yano S, Wayman G, et al. Cross talk between ERK and PKA is required for Ca2+ stimulation of CREB-dependent transcription and ERK nuclear translocation. Neuron. 1998;21(4):869-83.

63. Veyrac A, Besnard A, Caboche J, Davis S, Laroche S. The transcription factor Zif268/Egr1, brain plasticity, and memory. Prog Mol Biol Transl Sci. 2014;122: 89-129.

64. Duclot F, Kabbaj M. The role of early growth response 1 (EGR1) in brain plasticity and neuropsychiatric disorders. Front Behav Neurosci. 2017;11(35). https://www.frontiersin.org/articles/10.3389/fnbeh.2017.00035/full.

65. Choi SH, Bylykbashi E, Chatila ZK, Lee SW, Pulli B, Clemenson GD, et al. Combined adult neurogenesis and BDNF mimic exercise effects on cognition in an Alzheimer's mouse model. Science. 2018;1 (6406). https:// science.sciencemag.org/content/361/6406/eaan8821.long.

66. Shin J, Kong C, Cho JS, Lee J, Koh CS, Yoon MS, et al. Focused ultrasoundmediated noninvasive blood-brain barrier modulation: preclinical examination of efficacy and safety in various sonication parameters. Neurosurg Focus. 2018;44(2):E15.

\section{Publisher's Note}

Springer Nature remains neutral with regard to jurisdictional claims in published maps and institutional affiliations.

Ready to submit your research? Choose BMC and benefit from:

- fast, convenient online submission

- thorough peer review by experienced researchers in your field

- rapid publication on acceptance

- support for research data, including large and complex data types

- gold Open Access which fosters wider collaboration and increased citations

- maximum visibility for your research: over $100 \mathrm{M}$ website views per year

At BMC, research is always in progress.

Learn more biomedcentral.com/submissions 